\title{
Diacronie
}

Studi di Storia Contemporanea

$N^{\circ} 36,4 \mid 2018$

Viaggi e turismo nell'Europa del Novecento

\section{Andrea Miccichè, La Sicilia e gli anni Cinquanta. Il decennio dell'autonomia}

\section{Marco Grifo}

\section{OpenEdition}

Journals

\section{Edizione digitale}

URL: https://journals.openedition.org/diacronie/10483

DOI: 10.4000/diacronie. 10483

ISSN: 2038-0925

Editore

Association culturelle Diacronie

Notizia bibliografica digitale

Marco Grifo, «Andrea Miccichè, La Sicilia e gli anni Cinquanta. I/ decennio dell'autonomia», Diacronie [Online], N³6, 4 | 2018, documento 16, online dal 29 décembre 2018, consultato il 17 février 2022. URL: http://journals.openedition.org/diacronie/10483 ; DOI: https://doi.org/10.4000/diacronie.10483 


\section{Diacronie}

Studi di Storia Contemporanea

36, 4/2018

Viaggi e turismo nell'Europa del Novecento

\section{RECENSIONE: Andrea MICCICHÈ, La Sicilia e gli anni Cinquanta. Il decennio dell'autonomia, Milano, Franco Angeli, 2017, 262 pp.}

\section{A cura di Marco GRIFO}

Per citare questo articolo:

GRIFO, Marco, «RECENSIONE: Andrea MICCICHÈ, La Sicilia e gli anni Cinquanta. Il decennio dell'autonomia, Milano, Franco Angeli, 2017, 262 pp.», Diacronie. Studi di Storia Contemporanea : Viaggi e turismo nell'Europa del Novecento, 36, 4/2018, 29/12/2018,

URL: < http://www.studistorici.com/2018/12/29/grifo_numero_36/ >

Diacronie Studi di Storia Contemporanea $\rightarrow$ http://www.diacronie.it Rivista storica online. Uscita trimestrale.

redazione.diacronie@hotmail.it

Comitato di direzione: Naor Ben-Yehoyada - João Fábio Bertonha - Christopher Denis-Delacour - Maximiliano Fuentes Codera Anders Granås Kjøstvedt - John Paul Newman - Deborah Paci - Niccolò Pianciola - Spyridon Ploumidis - Wilko Graf Von Hardenberg

Comitato di redazione: Jacopo Bassi - Luca Bufarale - Gianluca Canè - Luca G. Manenti - Fausto Pietrancosta - Alessandro Salvador - Matteo Tomasoni - Luca Zuccolo citazione di estratti è comunque sempre autorizzata, nei limiti previsti dalla legge. 


\title{
16/ RECENSIONE: Andrea MICCICHÈ, La Sicilia e gli anni Cinquanta. Il decennio dell'autonomia, Milano, Franco Angeli, 2017, $262 \mathrm{pp}$.
}

\author{
A cura di Marco GRIFO
}

Visitammo molti cortili (sono specie di slarghi attorno a cui sono costruite delle catapecchie): i contadini e le donne dalle soglie ci facevano cenno di entrare perché vedessimo in che mondo vivevano. Per terra, nelle strade, nei cortili in pendio scorrono, per mancanza di fogne, le acque putride e il tanfo prende alla gola. Le case, se così si possono chiamare, sono delle tane dove piove dai tetti di canne, affumicate, spoglie, senza finestre, dove in pochi metri quadrati vivono accatastate otto, dodici persone ${ }^{1}$.

Così Carlo Levi descriveva la Sicilia durante il suo viaggio nel 1955. Sono atmosfere non molto diverse da quelle narrate negli stessi anni dalle inchieste di Danilo Dolci, Leonardo Sciascia e del giornale di Palermo «L'Ora»². Eppure, la Settimana Incom del 6 aprile 1955 descriveva una Sicilia in cammino: immagini di piazze, scuole, giardini, grattacieli in costruzione facevano da sfondo ad una terra «ringiovanita da sette anni di governo regionale», che «in pochi anni quintuplicava la produzione» ${ }^{3}$. L'isola diventava dunque il simbolo della rinascita economica e sociale dell'intero Paese.

È a partire da questa contrapposizione narrativa che si apre il libro di Andrea Miccichè La Sicilia e gli anni Cinquanta. Il decennio dell'Autonomia. L'opera, il cui titolo è un tributo al bel volume di Renée Rochefort ${ }^{4}$ del 1961, ricostruisce un'importante stagione della storia siciliana: quella dell'autonomismo. Andra Miccichè, che in alcuni saggi precedenti aveva già affrontato

\footnotetext{
${ }^{1}$ LEVI, Carlo, Le parole sono pietre. Tre giornate in Sicilia, Torino, Einaudi, 1955, pp. 109-113.

${ }^{2}$ DOLCI, Danilo, Banditi a Partinico, Bari, Laterza, 1955; SCIASCIA, Leonardo, Le parrocchie di Regalpetra, Bari, Laterza, 1956.

${ }^{3}$ SETTIMANA INCOM, Sicilia in Cammino, Istituto Luce, 4 aprile 1955, URL:

< https://www.youtube.com/watch?v=6Zh6O7OG49Y > [consultato il 30 ottobre 2018].

${ }^{4}$ ROCHEFORT, Renée, Le Travail en Sicile. Étude de géographie sociale, Paris, PUF, 1961.
} 
l'argomento 5 , si è occupato principalmente di storia della Spagna contemporanea, dedicando particolare attenzione ai temi del nazionalismo e del socialismo basco ${ }^{6}$.

Il lavoro, organizzato in quattro capitoli, più un prologo, un epilogo e un'appendice ripercorre la storia della Sicilia dal dopoguerra al 1959. Il punto di partenza è il secondo dopoguerra, quando l'opzione autonomistica maturava nel quadro del drammatico processo di transizione alla democrazia (1943-1945). In una situazione di miseria e di vuoto politico, una fetta della borghesia siciliana si era attestata su posizioni che prevedevano la separazione dell'isola dal resto del Paese. Il nazionalismo isolano, pieno di riferimenti alla depredazione subita dall'isola da parte degli stranieri (italiani compresi), si rivelava un efficace catalizzatore politico e faceva da collante a un personale politico eterogeneo. Nella compagine separatista spiccavano esponenti del blocco agrario, della borghesia dei mestieri e pezzi di una vecchia classe politica prefascista. Il separatismo, però, non ebbe vita facile e non sopravvisse alle sue ambiguità e alla ripresa di un sistema di partiti forte ed efficiente. Del separatismo rimase allora un "questione siciliana" a cui dare una risposta, «che in quel momento appariva già inscindibile dal problema della ricostituzione delle strutture dello Stato nazionale»7.

Lo Statuto di autonomia, fu voluto e difeso, anche se in modo diverso, da tutti i partiti del Comitato di Liberazione Nazionale e aveva individuato nei supposti torti subiti dallo Stato la rivendicazione storica alla base delle aspirazione di autogoverno. Non vi erano identità linguistiche, culturali da far rispettare ma solamente l'aspirazione a stimolare l'economia e ridurre il divario esistente con il resto del Paese. Utilizzando le parole dell'autore, «lo Statuto siciliano non era un mezzo di emancipazione identitaria», bensì "uno strumento utile a integrare la Sicilia a un centro economicamente e socialmente progredito» ${ }^{8}$.

È dunque all'interno di questa sfida tra centro e periferia che le forze politiche regionali si divisero, più che sulle consuete fratture politiche legate alla guerra fredda. Le narrazioni alternative con cui veniva descritta la Sicilia sono dunque da leggere all'interno della grande

\footnotetext{
${ }^{5}$ MICCICHÈ, Andrea, «Milazzismo autonomismo e crisi dei partiti nella Sicilia degli anni cinquanta», in Polo Sud, II, 3, 2013, pp. 83-110; ID., Auge e fine del milazzismo: l'autonomismo ed i partiti nella IV legislatura, in MICCICHÈ, Andrea, BOSCARELLO, Giuseppe (a cura di), Silvio Milazzo. L'attività parlamentare all'ARS, Firenze, Edit, 2013, pp. 75-94; ID, «Sicilia "all'addritta". Le elezioni del 1959, l'autonomismo e le sue narrazioni», in Meridiana, 82, 2015, pp. 135-144; ID, “"Sicilia in cammino”. Documentari, narrazioni e immagini di una società tra arretratezza e sviluppo (1953-1962)», in Officina della Storia, 17, 2017, URL:

<https://www.officinadellastoria.eu/it/2017/10/17/sicilia-in-cammino-documentari-narrazioni-eimmagini-di-una-societa-tra-arretratezza-e-sviluppo-1953-1962/ > [consultato il 30 ottobre 2018].

${ }^{6}$ Tra le altre citiamo: MICCICHÈ, Andrea, «Nazionalismo ed immigrazione: il caso basco», in Il Ponte, 5-6/ 2012, pp. 92-111; ID., «Lingua, razza ed evoluzione dell'identità basca. Come cambia un nazionalismo: il caso del Partido Nacionalista Vasco", in Spagna Contemporanea, 41, 2012, pp. 79-98; ID., De la clandestinidad a la actividad parlamentaria. El socialismo vasco y los primeros años de autonomía, 1973-1984, in MATEOS LÒPEZ, Abdon, LEÓN, Guillermo (a cura di), La reconstrucción del PSOE durante la Transición. Una perspectiva territorial, Madrid, Uned, 2017, pp. 91-104.

${ }^{7}$ MICCICHÈ, Andrea, La Sicilia e gli anni Cinquanta. Il decennio dell'autonomia, Milano, Franco Angeli, 2017, p. 23.

${ }^{8}$ Ibidem, p. 8.
} 
narrazione che i partiti fecero dell'autonomia: da una parte «l'autonomia delle realizzazione» della Democrazia cristiana e dall'altra «l'autonomia da realizzare» del Partito comunista. L'autonomia della Dc era quella di chi governava e metteva in mostra i propri successi e risultati materiali. Quella del Pci e del Psi era invece un'autonomia che difendeva gli interessi di una Sicilia sempre sfruttata e che rivendicava il suo ruolo in nome di un'autonomia tradita dai partiti di governo. Intorno all'autonomia, che era dunque la cifra distintiva della politica regionale di quegli anni, l'autore delinea i protagonisti e tre momenti principali (corrispondenti ai capitoli 1, 2 e 3).

I protagonisti della stagione autonomista furono trasversali a tutti i partiti: Giuseppe Alessi, Salvatore Aldisio, Silvio Milazzo, Giuseppe La Loggia, Franco Restivo della Democrazia Cristiana, Girolamo Li Causi ed Emanuele Macaluso del Partito Comunista. L'arco cronologico è diviso in tre momenti. Se il primo corrisponde alla creazione dell'apparato amministrativo della Regione e alla formazione di enti come l'Ente Siciliano Elettricità (1947); il secondo momento vede il dibattito sulla riforma agraria, sui progetti d'industrializzazione e la scoperta del petrolio a Ragusa. Il terzo e ultimo momento è quello che si sviluppò intorno all'elezioni del 1955, la cui campagna elettorale è concentrata sui temi dell'autonomia e dell'industrializzazione.

Una parte importante del libro è dedicata all'esperimento milazziano. Silvio Milazzo, esponente della Dc di Caltagirone e dell'autonomismo più radicale, venne eletto presidente della Regione siciliana con i voti, all'Assemblea regionale, dei partiti di sinistra e di destra, contro Giuseppe La Loggia, il candidato scelto dal suo partito e indicato dai vertici nazionali della Dc, allora guidata da Amintore Fanfani. La creazione, da parte di Milazzo, dell'Unione Siciliana Cristiano Sociale e il suo esperimento autonomista sono letti dall'autore come «l'episodio finale di una vicenda durata un decennio, il momento in cui le contraddizioni della politica siciliana si erano rivelate in maniera più compiuta». L'ipotesi di un partito autonomista era risultata impossibile e l'Uscs aveva finito per rappresentare solamente «le polemiche fazionarie locali, le ambizioni individuali di una parte della classe politica, il desiderio di riscatto di minoranze in uscita dalla Dc»9. Senza un reale progetto politico, la formazione autonomista di Milazzo si sfaldava al venir meno delle risorse che il governo regionale garantiva.

Il libro si chiude con l'elezione del 1959, che per la prima volta mandava la Dc all'opposizione ma dimostrava anche la debolezza dell'esperimento milazziano. Nel febbraio del 1960, infatti, uno scandalo relativo alla compravendita di un senatore portava alla conclusione di quell'esperienza. Si lasciava spazio ad un governo di centro-destra presieduto da Benedetto Majorana della Nicchiara, secondo «una formula che anticipava quella del governo Tambroni». Finiva dunque una stagione eccezionale della politica isolana e svaniva lentamente anche quella classe dirigente che ne era stata protagonista. Negli anni più recenti si avranno dei piccoli e contingenti ritorni di

${ }^{9}$ Ibidem, p. 233. 
fiamma, con Piersanti Mattarella, con Rico Nicolosi e Raffaele Lombardo che «dal passato riprendeva solo una retorica sicilianista che attribuiva ogni colpa allo Stato centrale», dimenticando le responsabilità della classe politica siciliana. Oggi, secondo l'autore, dell'autonomia resta

solo l'ormai annoso dibattito sulla sua crisi, alimentato da una discussione pubblica di tanto in tanto animata da giornalisti, opinionisti, pezzi di classe dirigente, che hanno frequentemente individuato nello Statuto la responsabilità dei mali della Sicilia e proposto la sua abrogazione come una sorta di panacea in grado di risolvere tutti i problemi di un'isola ${ }^{10}$.

Il merito del libro sta innanzitutto nell'aver ricostruito la stagione dell'autonomia siciliana, dimostrando la pluralità di significati che questa poteva assumere per i diversi partiti e restituendo a quest'ultima la sua funzione di motore della politica regionale. Un'accurata ricerca, fondata sullintreccio di fonti archivistiche e secondarie, apporta il suo contributo ad una storiografia ormai datata. Gli studi più recenti si erano interessati solamente in parte dell'autonomia, finendo per concentrarsi su aspetti come la criminalità organizzata, il separatismo e le lotte contadine. Inoltre gli studi sul nazionalismo basco hanno permesso all'autore una riflessione comparativa tra i due contesti analizzati. Da una parte una regione ricca e un nazionalismo etnoculturale e dall'altro una regione povera e un autonomismo riparazionista e un diverso rendimento delle istituzioni regionali rispetto quelle centrali. Come spiega l'autore «oggi è soprattutto il successo di tanti autonomismi europei e il loro buono stato di salute, anche in anni di crisi come quelli attuali, a rendere ancora più evidenti i fallimenti della Regione Sicilia» ${ }^{11}$.

\footnotetext{
${ }^{10}$ Ibidem, p. 235.

${ }^{11}$ Ibidem, p. 13.
} 


\section{L'AUTORE}

Marco GRIFO svolge il dottorato in Studi Storici presso le università degli studi di Firenze e di Siena, con una ricerca sul network di Danilo Dolci. Laureato in Storia presso l'Università degli Studi di Palermo e specializzato in Storia e Società presso l'Università degli Studi Roma Tre con il massimo dei voti. Ha conseguito il diploma presso l'Alta Scuola Roma Tre (ASTRE). Grazie a una borsa di mobilità d'Ateneo, ha svolto ricerca presso la Public Library e il LaGuardia College di New York. Ha collaborato con l'Istituto Luigi Sturzo per la realizzazione del progetto "La storia in rete. La stagione dei movimenti: 1965-1978".

URL: < http://www.studistorici.com/progett/autori/\#Grifo > 